\title{
Detection of glaucomatous damage in patients with osteo-odontokeratoprosthesis
}

Department of

Ophthalmology,

S Camillo General

Hospital, Rome, Italy

G C Falcinelli

$M$ Taloni

G Falcinelli

Eye Clinic, Catholic

University, Rome,

Italy

B Falsini

M Piccardi

Correspondence to:

Benedetto Falsini, MD, Eye

Clinic, Catholic University of Sacred Heart, Lgo F Vito 1 , I-00168, Rome, Italy.

Accepted for publication

15 September 1994

\begin{abstract}
Background-Osteo-odontokeratoprosthesis (OOKP) is an autologous transplantation procedure in which the cornea is replaced by an optical cylinder glued to a biological support. Patients undergoing OOKP surgery may develop a secondary glaucoma whose diagnosis, by means of standard diagnostic procedures, is often doubtful.
\end{abstract}

Methods-In the present study pattern electroretinograms (PERGs), visual evoked potentials (VEPs), contrast sensitivity, and automated threshold perimetry (Humphrey 30-2) were evaluated in 19 OOKP treated patients with postoperative visual acuities $\geqslant 0 \cdot 8$. Nine patients had had a preoperative secondary glaucoma, while the remaining 10 had no history of glaucoma and normal posterior pole.

Results-Results were compared with those obtained from either normal control subjects or from ordinary glaucoma patients. PERG amplitudes and contrast and perimetric sensitivities were reduced in both groups of OOKP patients when compared with normal controls. However, these losses were significantly greater in OOKP patients with glaucoma compared with those with normal posterior pole. VEPs were reduced, compared with controls, only in OOKP patients with glaucoma. These VEP losses were similar to those found in ordinary glaucoma patients.

Conclusion-Among the tests employed, VEPs showed the best accuracy $(79 \%)$ in discriminating between glaucomatous and non-glaucomatous OOKP treated eyes. The present results suggest a possible use of the VEP technique for detecting glaucomatous dysfunction after OOKP.

(Br f Ophthalmol 1995; 79: 129-134)

Osteo-odontokeratoprosthesis (OOKP) is an eterotopic autograft employed for treatment of severe corneal opacities not suitable for corneal transplantation. ${ }^{1}$ The cornea is replaced by an optical polymethylmethacrylate (PMMA) cylinder glued to a biological support (haptics), made by human living tissue (that is, an osteodental lamina). ${ }^{1-4}$ The OOKP technique has been successfully employed in large series of patients. ${ }^{5-7}$ Long term clinical ${ }^{7}$ and histological $^{8}$ results have suggested a greater stability and safety of OOKP compared with other types of keratoprostheses, whether with biological or biocompatible haptics. Recent innovative changes to the original Strampelli's technique,${ }^{7}$ have further improved the quality of anatomical and functional results, reducing the incidence of failures to less than $10 \%$ of cases.

Postoperative visual acuity has been considered the major functional parameter to evaluate the OOKP's rate of success. ${ }^{35-7}$ However, it must be taken into account that a considerable number of patients undergoing OOKP surgery might suffer from secondary glaucoma. ${ }^{7}$ The development or progression of this neuro-ophthalmic complication, which is very common in eyes with physical or chemical burns, may not affect visual acuity until advanced stages are reached, and may raise important diagnostic problems, mainly related to the difficulty in obtaining reliable clinical measures (that is, IOP, visual field) from OOKP treated eyes.

In recent years, refined electrophysiological (pattern evoked retinal and cortical potentials, PERG, VEPs) and psychophysical (contrast sensitivity, automated threshold perimetry within the central 30 degrees) tests have been employed to detect the development and progression of glaucomatous disease (see BodisWollner ${ }^{9}$ for a review). These methods can provide objective (evoked potentials) or subjective (psychophysical tests) measures of visual function that appear to be more sensitive and reliable than standard clinical tests. The use of these techniques could be of clinical value in identifying those OOKP treated patients suffering from concomitant secondary glaucoma.

In the present study we employed PERG, VEPs, contrast sensitivity, and automated perimetry to evaluate postoperatively visual function in OOKP treated patients with a recovery of Snellen acuity to normal or near to normal values. A subgroup of these patients had had a clinical history of secondary glaucoma before surgery. The results showed that both electrophysiological and psychophysical tests were able to differentiate OOKP treated patients with glaucomatous dysfunction from those with normal visual function. Among the functional tests employed, VEPs appeared to yield the best clinical discrimination between these two categories.

\section{Materials and methods}

\section{SUBJECTS}

Nineteen patients (12 males, seven females), who had been operated on for OOKP between 1988 and 1992 in the department of ophthalmology at S Camillo General Hospital (Rome, Italy), were included in the study. The 


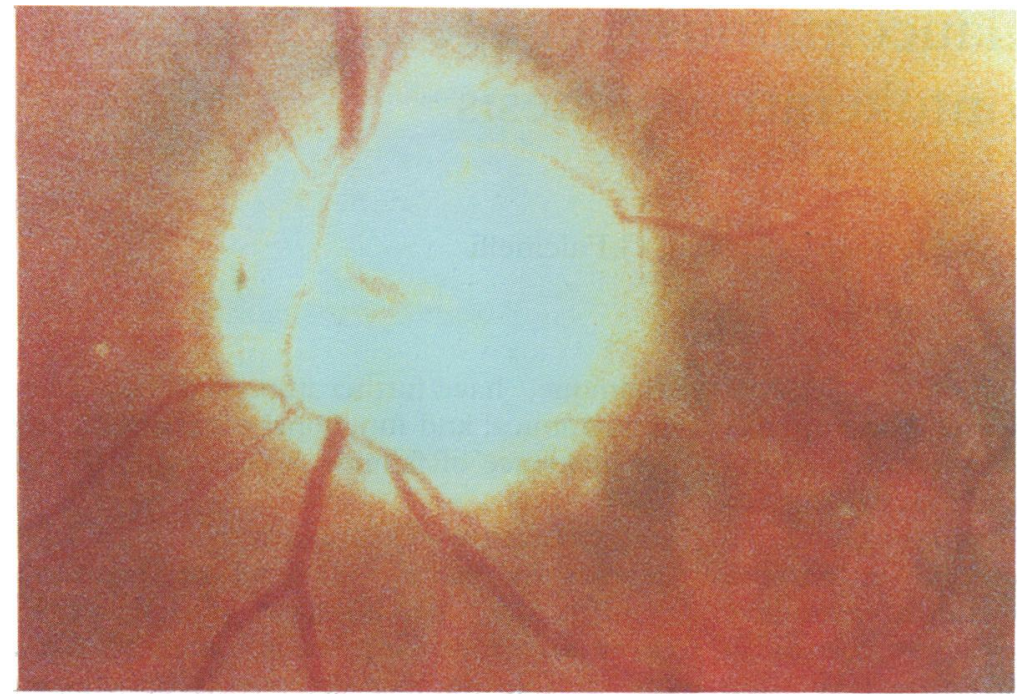

Figure 1 Representative example of an optic disc photograph taken from the right eye (through the keratoprosthesis) of one of the OOKP treated patients with a secondary glaucoma. Note the typical glaucomatous changes of the optic nerve (large cupping, reduced neuroretinal rim area). at the time of examination. Postoperative corrected visual acuity ranged from 0.8 to $1 \cdot 0$. Refractive errors were carefully corrected for the test recordings. All patients underwent electrophysiological and psychophysical tests at least 2 months after surgery.

Seventeen normal subjects (17 eyes), with an age and sex distribution comparable with that of the patients, were tested to obtain control data for PERG, VEP, and contrast sensitivity measurements. For comparison purposes with OOKP patients affected by a secondary glaucoma, eight patients with chronic open angle glaucoma were also evaluated. Their mean age was 48 (range 30-62) years. All these patients had typical optic disc and field defects, and were being treated with timolol maleate. None of them had undergone antiglaucomatous surgery.

\section{APPARATUS AND PROCEDURE}

\section{PERG, VEPs, and contrast sensitivity}

Visual stimuli employed for both electrophysiological recordings and contrast sensitivity measurements were vertical sinusoidal gratings of variable spatial frequency (between 0.6 and $4.8 \mathrm{c} / \mathrm{deg}$ ), electronically generated on a high resolution (Mangoni, Pisa-I) TV monitor $\left(24 \times 14 \mathrm{~cm}, 85 \mathrm{~cd} / \mathrm{m}^{2}\right.$ mean luminance) and square wave modulated in counterphase at $8 \mathrm{~Hz}$ (16 reversals/second). For PERG and VEP recordings the contrast of the stimulus was maintained at $56 \%$. For contrast sensitivity measurements a logarithmic attenuator was used to vary the contrast (in 1-10 dB steps) on the screen. Both patients and control subjects fixated monocularly at the centre of the stimulator from a distance of $57 \mathrm{~cm}$. Patients viewed the stimulus through the optical PMMA cylinder, whose size was $3.5 \mathrm{~mm}$. Control subjects were tested while wearing an artificial pupil of $3.5 \mathrm{~mm}$, in order to match their stimulus retinal illuminance with that of patients.

PERGs were recorded by a small silversilver chloride electrode taped over the skin of the lower eyelid. An equal electrode, placed over the eyelid of the contralateral, light tight patched eye, was used as reference (interocular ERG). With the same electrodes used for the PERG recordings, flash electroretinograms (to scotopic and photopic stimulation $^{12}$ ) were also recorded in all patients and controls. In all OOKP treated patients, the amplitudes and implicit times of flash electroretinograms were comparable with those obtained in control subjects. VEPs were recorded by an active silver-silver chloride electrode placed on the midline $2 \mathrm{~cm}$ above the inion, and referenced to the right mastoid. The left mastoid was grounded. Retinal and cortical signals were band pass filtered between 1 and $30 \mathrm{~Hz}$ ( $-6 \mathrm{~dB}$ per octave), amplified (100 000 fold), and averaged (12 bit resolution, $0.5 \mathrm{~ms}$ sampling rate, 250 events) with artefact rejection. Discrete Fourier analysis of the resulting waveforms was performed off line in order to isolate the the optic nerve. optic disc photograph, taken from the right eye of one of our OOKP treated patients, is reported in Figure 1.

None of the patients had vitreous opacities 

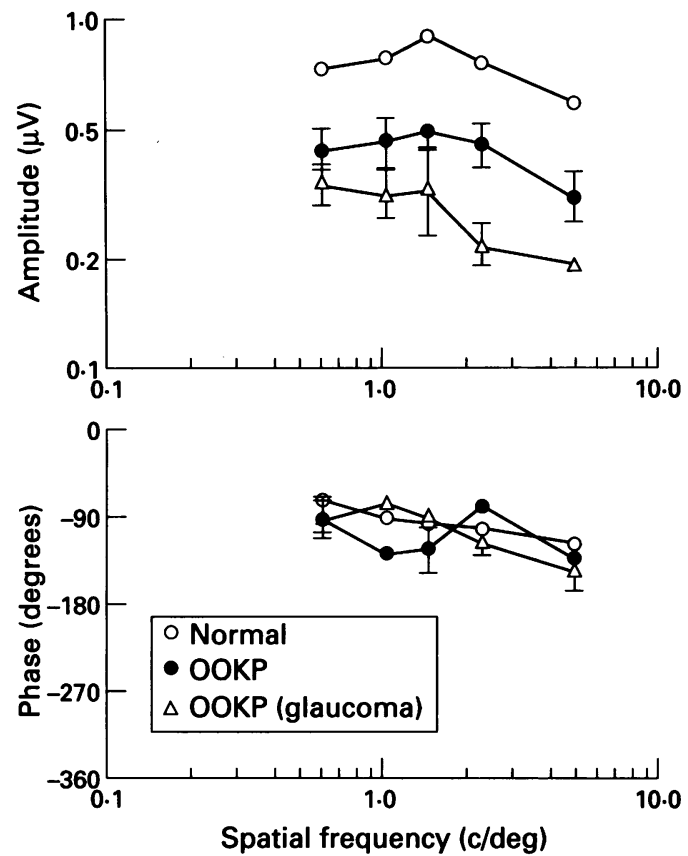

Figure 2 Mean (SEM) PERG amplitude and phase values as a function of spatial frequency for normal subjects, OOKP treated patients with normal posterior pole (OOKP), and OOKP treated patients with glaucoma (OOKP glaucoma).

component at the reversal frequency $(16 \mathrm{~Hz}$; second harmonic), whose amplitude $(\mu \mathrm{V})$ and phase (degrees) were measured and plotted as a function of spatial frequency. The mean of at least two records was taken at each spatial frequency. The average biological noise at the second harmonic, obtained with the monitor blanked at the same mean luminance as the stimulus, was $0.09 \mu \mathrm{V}$ for PERG and $0.2 \mu \mathrm{V}$ for VEP in both normal subjects and patients.

Contrast sensitivity was measured in the same experimental session as the electrophysiological recordings. The method of
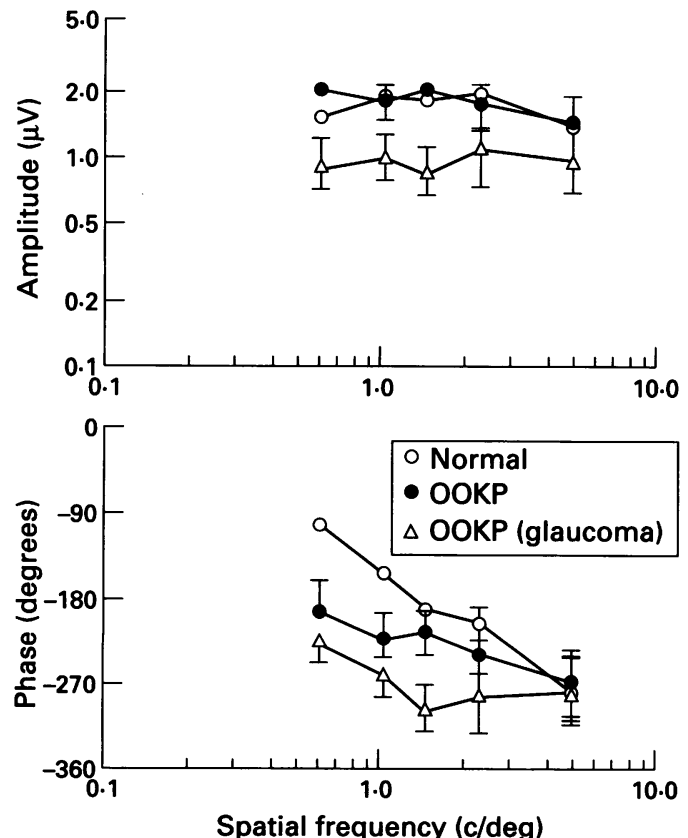

Figure 3 Mean (SEM) VEP amplitude and phase values as a function of spatial frequency for normal subjects, OOKP treated patients with normal posterior pole $(O O K P)$, and $O O K P$ treated patients with glaucoma (OOKP glaucoma). adjustment ${ }^{13}$ was used to determine contrast sensitivity. Three threshold estimates were taken for each spatial frequency. The average variation of the settings did not exceed $1.5 \mathrm{~dB}$ and was similar in the controls and patients.

PERG, VEPs, and contrast sensitivity data obtained from patients and controls were statistically evaluated by analysis of variance (ANOVA) with post hoc adjusted $t$ tests. The diagnostic sensitivity and specificity of the electrophysiological and psychophysical tests was evaluated, in the two groups of OOKP treated patients, by $2 \times 2$ contingency tables and Fisher's exact test.

\section{Automated perimetry}

Perimetric thresholds within central 30 degrees were evaluated by the Humphrey 30-2 threshold test. At least three measurements (on three separate days) were obtained from each patient. The results of the third test were considered for the purposes of the study. Perimetric indices (mean deviation (MD) corrected pattern standard deviation (CPSD) and short term fluctuation (SF)) were calculated with STATPAC software and compared with age and pupil size matched normative values. In addition, mean values of perimetric indices were compared between the OOKP patients' groups (that is, those with normal posterior pole and those with glaucoma) by unpaired $t$ tests. A qualitative field evaluation for glaucomatous defects was performed following the criteria proposed by Lustgarten et al. $^{14}$

\section{Results}

Figure 2 shows the mean (SEM) PERG amplitude and phase values as a function of spatial frequency for normal subjects, OOKP treated patients with normal posterior pole (OOKP), and OOKP patients with glaucoma (OOKP glaucoma). Mean PERG amplitudes showed a significant variation across groups (ANOVA, $F$ 37.8; df 2, 33; p <0.001), being reduced in patients in comparison with controls. Post hoc $t$ test revealed significant differences, pooled across spatial frequencies, between normal subjects and both groups of patients $(\mathrm{p}<0.001)$, and between OOKP patients with normal posterior pole and those with glaucoma $(p<0.05)$. Mean PERG phases did not change significantly across groups (ANOVA, F 1.33; df 2, 33; $p$ not significant).

Figure 3 shows the mean (SEM) VEP amplitudes and phases as a function of spatial frequency for normal subjects, OOKP treated patients with normal posterior pole, and OOKP treated patients with glaucoma. Mean VEP amplitudes changed significantly across groups (ANOVA, F 3.99; df 2, 33; p<0.05), being reduced in OOKP patients with glaucoma compared with the two other groups. Post hoc $t$ test revealed significant differences, pooled across spatial frequencies, between normal subjects and OOKP patients with glaucoma $(p<0.05)$, and between OOKP patients with normal posterior pole and those 


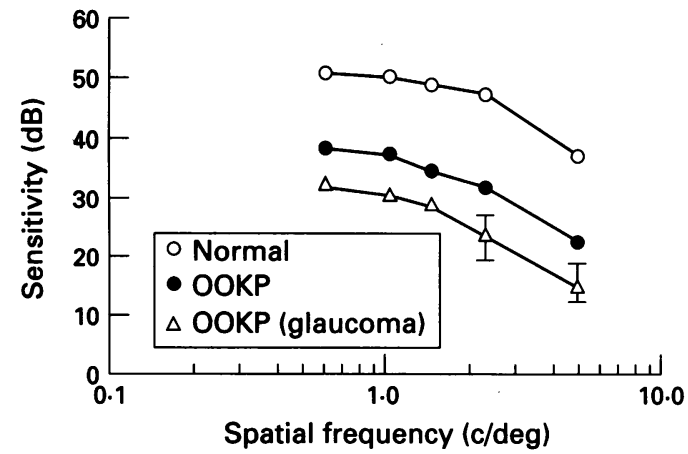

Figure 4 Mean (SEM) contrast sensitivity values (expressed in $d B$ of attenuation from the maximum stimulus contrast available) for normal subjects, $O O K P$ treated patients with normal posterior pole (OOKP), and OOKP treated patients with glaucoma (OOKP glaucoma).

with glaucoma $(p<0 \cdot 05)$. No significant differences were found between normal subjects and OOKP patients with normal posterior pole. Mean VEP phases changed significantly across groups (ANOVA, F 6.36; df 2, 33; p<0.01), showing a trend to a delay in OOKP patients compared with normal subjects. Post hoc $t$ tests revealed significant differences only between normal subjects and OOKP patients with glaucoma $(p<0 \cdot 01)$. These differences were apparent at low medium but not at higher spatial frequencies (see Fig 3).

Figure 4 shows mean (SEM) contrast sensitivity values (expressed in $\mathrm{dB}$ of attenuation from the maximum stimulus contrast available) for normal subjects, OOKP treated patients with normal posterior pole, and OOKP treated patients with glaucoma. Mean contrast sensitivities changed significantly across groups (ANOVA, $F$ 55.5; df 2, 33; $\mathrm{p}<0.001$ ), being reduced in patients compared with controls. Post hoc $t$ test revealed significant differences, pooled across spatial frequencies, between normal subjects and both groups of patients $(p<0.001)$, and between OOKP patients with normal posterior pole and those with glaucoma $(p<0.05)$.

Table 1 reports the mean (SEM) values of perimetric indices recorded in OOKP treated patients with normal posterior pole and in those with glaucoma. In both groups of patients, the MD and CPSD were, on average, significantly different (lower and larger, respectively) than normal values. By contrast, the SF mean values were within the range of normal variability. Unpaired $t$ test showed that, on average, $\mathrm{MD}$ was significantly lower, and CPSD significantly larger, in OOKP patients with glaucoma compared with those with normal posterior pole $(p<0.05)$. Most of the OOKP treated patients (either with or

Table 1 Mean values (SD) of perimetric indices recorded in OOKP treated patients with normal posterior pole and in those with secondary glaucoma

\begin{tabular}{lrc}
\hline & \multicolumn{1}{c}{$O O K P$} & \multicolumn{1}{c}{ OOKP (glaucoma) } \\
\hline Mean deviation (dB) & $-14.9(1.9)$ & $-19.1(5.2)$ \\
Corrected pattern & $8.4(1.8)$ & $11.8(1.7)$ \\
standard deviation & $1.6(0.5)$ & $1.9(0.5)$ \\
Short fluctuation & \\
\hline
\end{tabular}
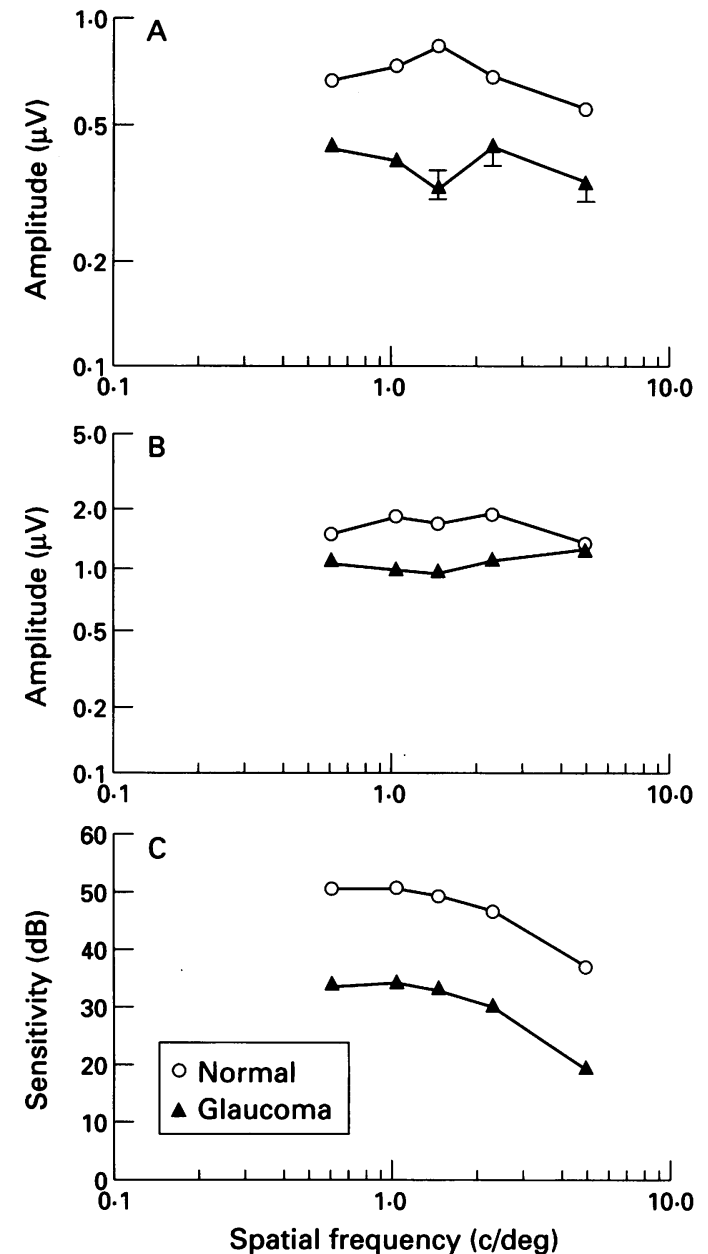

Figure 5 Mean (SEM) PERG (A) and VEP (B) amplitudes and contrast sensitivity (C) values, as a function of spatial frequency, for normal subjects and control glaucoma patients. Note (see Fig 3 for comparison) that VEP losses in glaucoma patients are similar to those of $O O K P$ treated patients with glaucoma and greater than those of OOKP patients with normal posterior pole.

without glaucoma) had a generalised depression of sensitivity across their visual fields, which made an assessment for localised glaucomatous defects difficult. However, in four out of the nine OOKP patients with glaucoma, a localised, typical glaucomatous defects (arcuate scotoma, nasal step, or paracentral scotoma) was found, while such defects were not observed in the OOKP patients with normal posterior pole.

Figure 5 shows the results obtained with PERG, VEPs, and contrast sensitivity (mean values (SEM)) in the control groups of chronic open angle glaucoma patients and normal subjects. When compared with normal subjects, glaucoma patients had significant reductions in the mean PERG $(F 28 \cdot 6$, df 1,23 , $\mathrm{p}<0.01)$ and VEP $(F 8.7$, df 1, 23, p <0.01) amplitudes, as well as in the mean contrast sensitivity values $(F 181 \cdot 8$, df $1,23, \mathrm{p}<0 \cdot 01)$. The PERG and contrast sensitivity losses found in the control glaucoma patients did not differ significantly from those of OOKP treated patients, either with normal posterior pole or with glaucoma (see, for comparison, Figs 2 and 4). By contrast, the VEP amplitude losses of the control glaucoma patients were significantly greater than those of OOKP treated patients with normal posterior pole, and similar to those 
of OOKP treated patients with glaucoma (see, for comparison, Fig 3).

Individual electrophysiological (PERG and VEPs amplitudes and phases) and psychophysical (contrast sensitivities and perimetric indices) results obtained from OOKP treated patients were analysed in order to determine which of the tests employed in this study was able to best discriminate between glaucomatous and non-glaucomatous patients. To this goal, the data obtained from each patient were compared with the corresponding $95 \%$ confidence limits (that is, plus or minus $2 \mathrm{SD}$ ) established for normal subjects. An individual patient's value was defined as abnormal when outside these limits. The number of abnormal results obtained with each test was then compared in non-glaucomatous and glaucomatous OOKP treated patients. We found that VEPs to $1.4 \mathrm{c} / \mathrm{deg}$ spatial frequency stimuli were abnormal (in amplitude, phase, or both) in one out of $10(10 \%)$ OOKP patients with normal posterior pole, and in six out of nine $(67 \%)$ OOKP patients with glaucoma. The difference between the two patient groups was statistically significant (Fisher's exact test: $p=0.02$ ), and corresponded to a $75 \%$ test sensitivity, $85 \%$ specificity, and $79 \%$ total accuracy. Other tests yielded poorer clinical discriminations (Fisher's exact test: p not significant) between the two patient groups. For both PERG and contrast sensitivity the best discriminating responses were those to $4.8 \mathrm{c} / \mathrm{deg}$ spatial frequency stimuli. With these responses, PERG had $61 \%$ test sensitivity, $66 \%$ specificity, and $63 \%$ total accuracy, while contrast sensitivity had $100 \%$ test sensitivity, but only $53 \%$ specificity and $57 \cdot 8 \%$ of total accuracy.

\section{Discussion}

A stable recovery in visual acuity to normal or near normal values $(0 \cdot 8-1 \cdot 0)$ is frequently reported in eyes that have received OOKP surgery for severe corneal opacities not treatable by perforating keratoplasty $(56.6 \%$ of 134 eyes in the series reported by Falcinelli et $a l^{7}$ ). However, a significant proportion of those OOKP treated eyes may develop a secondary glaucoma, which can be considered the most frequent complication in OOKP. ${ }^{7}$ Glaucoma may induce a subtle and progressive visual dysfunction that is not reflected, unless in advanced stages, by changes in visual acuity. Reliable and sensitive methods for glaucoma diagnosis in OOKP treated eyes are therefore needed. In this respect, standard clinical measures may not be adequate. IOP is difficult to measure reliably either in diseased corneas before surgery, or after OOKP has been implanted. Peripheral visual fields, determined by Goldmann kinetic perimetry, may be significantly affected by the relatively small diameter of the optical PMMA cylinder. ${ }^{15}$

In the present study, we evaluated the possible use of some tests of central visual functioning (PERGs, VEPs, contrast sensitivity, and automated perimetry) for detecting glaucomatous dysfunction in OOKP treated eyes. We tested two groups of those eyes, one with normal posterior pole and no history of glaucoma and the other with clinical signs and a history indicative of preoperative secondary glaucoma. The results from these two groups were also compared with those from unoperated control eyes, either normal or glaucomatous. We found that both electrophysiological and psychophysical tests were altered, compared with controls, in both normal and glaucomatous OOKP treated eyes. Indeed, PERG amplitudes as well as contrast and perimetric sensitivities were reduced, and VEP phases were delayed, in both groups of patients in comparison with controls. These changes may be at least in part related to a reduced optical quality (that is, reduced effective retinal size ${ }^{15}$ ) of visual stimuli viewed through the PMMA cylinder, whose length and refractive power are $7.2 \mathrm{~mm}$ and 55 dioptres, respectively. A reduction in the effective retinal contrast of visual stimuli may also have occurred in patients, because of possible changes in the eyes' modulation transfer functions induced by the optical PMMA prostheses. The large losses in psychophysical sensitivities (about $15 \mathrm{~dB}$ for both contrast sensitivity and automated perimetry) found in non-glaucomatous OOKP patients might give an indication of the amount of contrast attenuation through OOKP prosthesis. However, PERG, VEPs, contrast sensitivity, and perimetric losses were significantly more marked in OOKP operated eyes with glaucoma compared with those with normal posterior pole, indicating the presence of glaucoma related changes in both electrophysiological and psychophysical variables. This is also supported by the finding that, in the unoperated control eyes with glaucoma, PERG, VEP, and contrast sensitivity were reduced in comparison with normal eyes. Interestingly, VEP losses of glaucoma control eyes were similar to those found in OOKP treated eyes with glaucoma, and greater than those of OOKP eyes with normal posterior pole.

Among the tests employed in this study, we found that VEP responses to medium spatial frequencies $(1.4 \mathrm{c} / \mathrm{deg}$ ) yielded the best discrimination, in terms of sensitivity and specificity, between glaucomatous and nonglaucomatous OOKP eyes. Although this finding needs to be confirmed in a larger series of patients, it suggests that VEPs may have a specific clinical value for diagnosis of glaucomatous damage in OOKP treated patients.

Abnormalities in electrophysiological and psychophysical tests have been typically reported in previous functional studies on glaucoma. ${ }^{9141617}$ It is also known ${ }^{9}$ that these tests may be of clinical value for monitoring the progression of glaucomatous damage. This suggests a possible use of the techniques employed in the present study for postoperative longitudinal evaluation of glaucomatous OOKP patients. Preliminary results of an ongoing follow up study, which will be the object of a separate report, are encouraging in this respect.

In conclusion, our results indicate that both 
electrophysiological methods and psychophysical measures employed for glaucoma diagnosis might be helpful for the postoperative assessment of visual function in OOKP patients who are being suspected of having a secondary glaucoma. In clinical setting, VEPs appear to give the best sensitivity and specificity for detecting a glaucomatous dysfunction after OOKP.

1 Strampelli B. Tecnica e risultati dell'odonto cheratoprotesi. Atti XLV Congresso SOI 1964; 22: 288-91.

Strampelli B, Boyd B. Osteo-odontoprosthokeratoplasty. Highlights in ophthalmology. VS Editions 1967; 10: 212

3 Strampelli B. Osteo-odonto-keratoprothese. Ber Dtsch Ophthalmol Ges 1972; 71: 322-35.

4 Strampelli B. Simposio sulla cheratoprotesi. Atti LVII Congresso SOI 1976: 390-450.

5 Temprano J. Osteo-odonto-keratoprostheses: fifteen years of experience. In: Khoo CY, et al, eds. New frontiers in ophthalmology. 1991: 532-8.

6 Falcinelli G, Missiroli A, Petitti V, Pinna C. Osteo-odontokeratoprosthesis up-to-date. Acta $X X V$ Concilium Ophthalmologicum. Milan: Kugler and Ghedini, 1987; 2: 2772-6.

7 Falcinelli GC, Barogi G, Taloni M, Falcinelli G. Osteodontokeratoprosthesis: present experience and Osteodontokeratoprosthesis: present experience
future prospects. Refract Corneal Surg 1993; 9: 193.

8 Ricci R, Pecorella I, Ciardi A, Della Rocca C, Di Tondo U,
Marchi V. Strampelli's osteoodontokeratoprosthesis Clinical and histological long-term features of three prostheses. Br f Ophthalmol 1992; 76: 232-4.

9 Bodis-Wollner I. Electrophysiological and psychophysical testing of vision in glaucoma. Surv Ophthalmol 1989; 33 (suppl): $301-7$.

10 Whitacre MM, Stein RA, Hassanein K. The effect of corneal thickness on applanation tonometry. $A m$ I Corneal thickness on applan

11 Klein BEK, Magli YL, Richie KA, Moss SE, Meuer SM Klein R. Quantitation of optic disc cupping. Ophthalmology 1985; 92: 1654-6.

12 Marmor MF, Ardeil GB, Nilsson SEG, Zrenner E Standard for clinical electroretinography: Arch Ophthalmo 1989; 107: 816-9.

13 Plant GT, Hess RF, Thomas SJ. The pattern evoked electroretinogram in optic neuritis. A combined psychophysical and electrophysiological study. Brain 1986; 109. physical 8 .

14 Lustgarten JS, Marx MS, Podos SM, Bodis-Wollner I, Campeas D, Serle JB. Contrast sensitivity and computerized perimetry in early detection of glaucomatous puterized perimetry in early detectio

15 Rol P, Parel JM, Lacombe E, Legeais JM, Villain F Optics of keratoprostheses. Refract Corneal Surg 1993; 9: 212-3.

16 Porciatti V, Falsini B, Brunori S, Colotto A, Moretti G. Pattern electroretinogram as a function of spatial frequency in ocular hypertension and early glaucoma. $D o c$ Ophthalmol 1987; 65: 349-55.

17 Marx MS, Podos SM, Bodis-Wollner I, Ping-You Lee, Ron-Fanf Wang, Severin C. Signs of early damage in glaucomatous monkey eyes: low spatial frequency losses in the pattern ERG and VEP. Exp Eye Res 1988; 46: in the pat 